\title{
Moment-norm gradient flow on flag manifolds
}

\author{
JOHN LORCH
}

\begin{abstract}
A geometric proof of the Matsuki orbit duality for flag manifolds is established in [R. Bremigan and J. Lorch, Orbit duality for flag manifolds, Manuscripta Math. 109 (2002), 233-261.] by analyzing the gradient flow of the norm-squared of a moment map. In the present paper, we investigate explicit formulas for integral curves associated with this flow, leading to a correspondence between certain integral curves and Cayley transforms. In addition, an exhaustive collection of curves is presented in the rank-one hermitian symmetric case.
\end{abstract}

\section{Introduction}

\subsection{Purpose}

One may obtain a geometric proof of the Matsuki orbit duality for flag manifolds by analyzing the gradient flow of a moment-norm function (see [2]). The purpose of this paper is to investigate explicit formulas for integral curves associated with this flow, leading to a correspondence between certain integral curves and Cayley transforms. In addition, an exhaustive collection of curves is presented in the rank-one hermitian symmetric case.

\subsection{Background and structure}

In its most basic formulation, Matsuki duality is a one-to-one correspondence between the $G_{0-}$ and $K$-orbits of a flag variety $X=G / Q$ (for notation, see Section 2.1). While the first proof [7] of the duality was algebraic in nature, we are concerned with the geometric proof given in [2] employing the moment-norm technique: Drawing motivation from $[5,8,10]$, one begins

1991 Mathematics Subject Classification Primary 22F30; Secondary 22E46, 14M15, 37C10 
with a function $f^{+}: X \rightarrow \mathbb{R}$ given by the norm-squared of a moment map $\mu: X \rightarrow \mathfrak{k}_{0}^{*}$ for an appropriate Kähler structure on $X$. Corresponding to $f^{+}$ are a critical set $\mathcal{C} \subset X$ and an extended gradient flow $\Gamma:[-\infty, \infty] \times X \rightarrow$ $X$, which satisfies $\Gamma(0, x)=x$ for all $x \in X$ and $\Gamma(t, c)=c$ for all $c \in \mathcal{C}$. Further, put $\pi^{ \pm}: X \rightarrow \mathcal{C}, \pi^{ \pm}(x):=\Gamma( \pm \infty, x)$. There are a finite number of $K_{0}$-orbits in the critical set $\mathcal{C}$, and the inverse image in $X$ under $\pi^{+}$ (resp. $\pi^{-}$) of a $K_{0^{-}}$orbit is a single $G_{0}$ (resp. $K$ ) orbit. Thus, there are two stratifications of $X$ coming from $\pi^{+}$and $\pi^{-}$which are indexed by the set of $K_{0}$-orbits in $\mathcal{C}$, and these stratifications coincide with the decompositions of $X$ into $G_{0^{-}}$and $K^{\prime}$-orbits, respectively. These ideas lead naturally to the Matsuki correspondence, with two orbits being in duality exactly when their intersection is a $K_{0}$-orbit of critical points for $f^{+}$.

The analysis in [2] did not require an explicit computation of integral curves for $\nabla f^{+}$. However, it is reasonable to hope that one may recover additional information about $X$ as a by-product of rendering these curves. Using $X=\mathrm{SL}(2, \mathbb{C}) / B$ for inspiration, we investigate curves of the form $\gamma(t)=\operatorname{Ad}\left(e^{s(t) Z}\right) x$, where $x$ is a critical point for $f^{+}$in $X$ (considered as an $\operatorname{Ad}\left(G_{u}\right)$-orbit in $\mathfrak{g}_{u}$ when convenient), $s(t)$ is a real-valued function and $Z \in \mathfrak{g}_{u}$ is tangent either to $G_{0} \cdot x$ or to $K \cdot x$ and is perpendicular to $K_{0} \cdot x$. We will see that such a curve $\gamma(t)$ is an integral curve for $\nabla f^{+}$if and only if $s(t)$ is a solution of a certain differential equation. In general, this approach yields integral curves and associated information about $X$ (e.g., Cayley transforms), but the method works best for irreducible rank-one hermitian symmetric spaces, in which case $\gamma(t)$ is an integral curve for every reasonable choice of "direction" $Z \in \mathfrak{g}_{u}$.

The paper is organized as follows: Section 2 contains notation and a brief review of results needed for later in paper. Candidates for integral curves and the differential equation determining the viability of a given curve are given in Sections 3 and 4, respectively. In Section 5 we see that certain integral curves for $\nabla f^{+}$correspond to Cayley transforms for $X$. Finally, in Section 6 we show that every appropriate $Z \in \mathfrak{g}_{u}$ gives rise to an integral curve for $\nabla f^{+}$in the case that $X$ is hermitian symmetric of rank one.

\section{Background material}

In the interest of self-contained exposition, we provide brief background information. The results in this section, accompanied by full technical details, may be found in $[2,4]$. 


\subsection{Notation}

Notation 2.1. (a) Let $G$ be a connected complex semisimple Lie group with Lie algebra $\mathfrak{g}$. Let $\kappa$ represent the complex bilinear Killing form on $\mathfrak{g}$.

(b) Let $\theta: G \rightarrow G$ be a Cartan involution. The group of fixed points $G_{u}:=$ $G^{\theta}$ is a maximal compact subgroup of $G$. We also write $\theta$ for the corresponding involution of $\mathfrak{g}$. We have a $G_{u}$-invariant norm-squared function on $\mathfrak{g}$ given by $Z \mapsto\|Z\|_{\kappa}^{2}:=-\kappa(Z, \theta(Z))$.

(c) Let $\sigma: G \rightarrow G$ be a complex conjugation commuting with $\theta$. The subgroup of fixed points $G_{0}:=G^{\sigma}$ is a real form of $G$.

(d) Let $K_{0}:=G_{0} \cap G_{u}$, a maximal compact subgroup of $G_{0}$; then $K:=G^{\sigma \theta}$ is the complexification of $K_{0}$ in $G$.

(e) Let $Q$ be a parabolic subgroup of $G$ possessing a $\sigma, \theta$-stable maximal torus $T$. Let $\mathfrak{t}=$ Lie $T$ and $\mathfrak{t}_{u}=\mathfrak{t} \cap \mathfrak{g}_{u}$.

(f) Write $Q=L \ltimes R_{\mathrm{U}} Q$, where $R_{\mathrm{U}} Q$ is the unipotent radical of $Q$, and $L$ is the Levi factor containing $T$.

(g) Let $\mathfrak{g}_{u}, \mathfrak{g}_{0}, \mathfrak{q}, \mathfrak{l}, \mathfrak{k}, \mathfrak{k}_{0}$ and $\mathfrak{r}$ represent the Lie algebras of $G_{u}, G_{0}, Q$, $L, K, K_{0}$ and $R_{\mathrm{U}} Q$, respectively.

(h) Let $X$ denote the variety of parabolic subgroups of $G$ that are conjugate to $Q$. Given $x \in X$, we write $Q_{x}$ and $\mathfrak{q}_{x}$ for the parabolic subgroup and subalgebra corresponding to $x$. Since $N_{G} Q=Q$, we may identify $X \simeq G / Q$

(i) Let $S$ be a maximal torus of a parabolic $Q_{x} \subset G$, with the unique Levi decomposition $Q_{x}=L_{x} \ltimes R_{U} Q_{x}$ such that $S \subset L_{x}$. Let $\Delta(\mathfrak{g}, \mathfrak{s})$ be the collection of roots of $\mathfrak{g}$ relative to $\mathfrak{s}=$ Lie $S$. Recall that we may choose a simple system $\Pi \subset \Delta(\mathfrak{g}, \mathfrak{s})$ and $\Pi^{\prime} \subset \Pi$ satisfying

$$
\mathfrak{l}_{x}=\mathfrak{s} \oplus \bigoplus_{\alpha \in \Delta_{\Pi^{\prime}}(\mathfrak{g}, \mathfrak{s})} \mathfrak{g}_{\alpha} \quad \text { and } \quad \mathfrak{r}_{x}=\bigoplus_{\alpha \in \Delta^{+}(\mathfrak{g}, \mathfrak{s}) \backslash \Delta_{\Pi^{\prime}}(\mathfrak{g}, \mathfrak{s})} \mathfrak{g}_{\alpha}
$$

where $\Delta_{\Pi^{\prime}}(\mathfrak{g}, \mathfrak{s})=\operatorname{Span}_{\mathbb{Z}}\left(\Pi^{\prime}\right) \cap \Delta(\mathfrak{g}, \mathfrak{s})$. We have identifications $\Delta_{\Pi^{\prime}}(\mathfrak{g}, \mathfrak{s})=\Delta\left(\mathfrak{l}_{x}, \mathfrak{s}\right)$ and $\Delta^{+}(\mathfrak{g}, \mathfrak{s}) \backslash \Delta_{\Pi^{\prime}}(\mathfrak{g}, \mathfrak{s})=\Delta\left(\mathfrak{r}_{x}, \mathfrak{s}\right)$.

(j) We write $\tilde{\mathfrak{r}}_{x}$ for $\theta \mathfrak{r}_{x}$, which is the Lie algebra of the unipotent radical of $Q_{x}^{\text {opp }}$, and $Z=Z_{\tilde{r}_{x}}+Z_{\mathfrak{l}_{x}}+Z_{\mathfrak{r}_{x}}$ to reflect the decomposition $\mathfrak{g}=\tilde{\mathfrak{r}}_{x} \oplus$ $\mathfrak{l}_{x} \oplus \mathfrak{r}_{x}$. 


\subsection{Flag manifolds and adjoint orbits}

The variety $X=G / Q$ in item (h) of Notation 2.1 is a flag variety. It is known that $X$ is a compact complex manifold, and that $G_{u}$ acts transitively on $X$ (see $[4,12]$ ). It is useful to identify $X$ with an orbit of $G_{u}$ in $\mathfrak{g}_{u}$. We recall the construction here.

Lemma 2.2. There exists an element $\Upsilon \in \mathfrak{t}_{u}$ such that $\alpha(\Upsilon)=0$ for $\alpha \in$ $\Delta(\mathfrak{l}, \mathfrak{t})$, and $\alpha(i \Upsilon)<0$ for $\alpha \in \Delta(\mathfrak{r}, \mathfrak{t})$.

Lemma 2.2 together with the fact that $X \simeq G / Q \simeq G_{u} /\left(G_{u} \cap L\right)$ gives

Proposition 2.3. Let $\Upsilon \in \mathfrak{t}_{u}$ be as in Lemma 2.2. We may identify $X$ with the $\operatorname{Ad}_{G_{u}}$-orbit of $\Upsilon$ in $\mathfrak{g}_{u}$ via $g Q g^{-1} \mapsto^{g} \Upsilon$ for $g \in G_{u}$.

Henceforth we frequently identify $x \in X$ with the corresponding point $\Upsilon_{x}$ in the $G_{u}$-orbit of $\Upsilon$ in $\mathfrak{g}_{u}$, which we denote $\mathcal{O}$. We identify $\mathfrak{g} / \mathfrak{q}_{x} \simeq$ $T_{x} X \simeq T_{\Upsilon_{x}} \mathcal{O} \simeq \mathfrak{g}_{u} /\left(\mathfrak{l}_{x} \cap \mathfrak{g}_{u}\right)$. Finally, we let $x_{0}$ denote the base point in $X$ corresponding to $Q$.

\subsection{Almost complex structure}

Observe that the mapping $I_{x}: \mathfrak{g} / \mathfrak{q}_{x} \rightarrow \mathfrak{g}_{u} / \mathfrak{g}_{u} \cap \mathfrak{l}_{x}$ defined by

$$
I_{x}\left(Z+\mathfrak{q}_{x}\right)=\left(Z_{\tilde{\mathfrak{r}}_{x}}+\theta Z_{\tilde{\mathfrak{r}}_{x}}\right)+\left(\mathfrak{g}_{u} \cap \mathfrak{l}_{x}\right)
$$

is the inverse of the mapping $\mathfrak{g}_{u} /\left(\mathfrak{g}_{u} \cap \mathfrak{l}_{x}\right) \rightarrow \mathfrak{g} / \mathfrak{q}_{x}$ induced by the inclusion $\mathfrak{g}_{u} \subset \mathfrak{g}$. This immediately yields an almost complex structure on $G_{u} /$ $\left(L_{x} \cap G_{u}\right) \simeq \mathcal{O}:$

$$
Z+\left(\mathfrak{l}_{x} \cap \mathfrak{g}_{u}\right)=Z_{\mathfrak{\mathfrak { r }}_{x}}+Z_{\mathfrak{r}_{x}}+\left(\mathfrak{l}_{x} \cap \mathfrak{g}_{u}\right)
$$

$$
\stackrel{J_{x}}{\longrightarrow} i Z_{\tilde{\mathfrak{r}}_{x}}-i \theta\left(Z_{\tilde{\mathfrak{r}}_{x}}\right)+\left(\mathfrak{l}_{x} \cap \mathfrak{g}_{u}\right)=i Z_{\tilde{\mathfrak{r}}_{x}}-i Z_{\mathfrak{r}_{x}}+\left(\mathfrak{l}_{x} \cap \mathfrak{g}_{u}\right) .
$$

\subsection{Kähler structure}

Recalling that any coadjoint orbit of a Lie group is a symplectic manifold, we may put a symplectic structure on $X \sim \mathcal{O}$ following the construction given in [1]. This structure, which turns out to be Kähler, is given by

$$
\left\langle\xi_{Z}, \xi_{W}\right\rangle_{x}=-2 i \kappa\left(\Upsilon_{x},\left[Z_{\tilde{\mathfrak{r}}_{x}}, \theta W_{\tilde{\mathfrak{r}}_{x}}\right]\right),
$$

where $Z, W \in \mathfrak{g}, x \in X$ and $\xi_{Z}$ denotes the vector field on $X$ induced by $Z$. 


\subsection{The moment-norm function $f^{+}$and its gradient}

We now define a real-valued, $K_{0}$-invariant function $f^{+}$on $X$ (the energy function of [4]) which is used in [2] to establish the Matsuki correspondence. Ingredients include the (dual) moment map $\mu_{G_{u}}: X \rightarrow \mathfrak{g}_{u}$, which is given by

$$
\mu_{G_{u}}(x)=\Upsilon_{x}
$$

together with the squared Killing norm:

Definition 2.4. Let $f^{+}: X \rightarrow \mathbf{R}$ be given by

$$
f^{+}(x)=2\left\|\operatorname{proj}_{\mathfrak{k}_{0}}^{\mathfrak{g}_{u}}\left(\mu_{G_{u}}(x)\right)\right\|_{\kappa}^{2}=2\left\|\mu_{K_{0}}(x)\right\|_{\kappa}^{2} .
$$

The gradient of $f^{+}$at $x \in X$ is

$$
\nabla f^{+}=-2 J \xi_{\sigma \mu_{G u}(x)}=-4 J \xi_{\mu_{K_{0}}(x)}
$$

In [2], the gradient flow to infinity of $f^{+}$plays a central role in the proof of Matsuki duality. Of particular importance are the various characterizations of the critical set for $f^{+}$(e.g., $x$ is a critical point if and only if $\mathfrak{l}_{x}$ contains a $\sigma, \theta$-stable Cartan subalgebra), as well as understanding the interaction between $\nabla f^{+}$and the tangent spaces to various orbits. Two results of this flavor that are relevant for this paper are:

Proposition 2.5. For all $x \in X, \nabla f^{ \pm}(x)$ is tangent to the $G_{0}$-orbit of $X$ and to the $K$-orbit of $x$.

Proposition 2.6. Let $x$ be a critical point for $f^{+}$. With respect to the real part of the Kähler form given in (2.3), the orthogonal complement of $T_{x}\left(K_{0} \cdot x\right)$ in $T_{x}\left(G_{0} \cdot x\right)$ is isomorphic to ad ${ }_{i \Upsilon_{x}}^{-1}\left(\tilde{\mathfrak{r}}_{x}^{-\sigma \theta}\right)$, while the orthogonal complement of $T_{x}\left(K_{0} \cdot x\right)$ in $T_{x}(K \cdot x)$ is isomorphic to $\operatorname{ad}_{i \Upsilon_{x}}^{-1}\left(\tilde{\mathfrak{r}}_{x}^{-\sigma}\right)$. Further, these two complements are orthogonal to each other.

\section{A candidate for the flow}

Recall that our main purpose is to produce explicit integral curves for $\nabla f^{+}$. In this section, we present candidates for these curves. We will assume the base point $x_{0}$ for $X$ is a critical point for $f^{+}$, and we let $\Upsilon \in \mathfrak{g}_{u}$ represent the corresponding point in $\mathcal{O}$. We begin with two observations. 
Proposition 3.1. For each $x \in X, \nabla f^{+}(x)$ lies in the orthogonal complement of $T_{x}\left(K_{0} \cdot x\right)$.

Proof. Let $W \in T_{x}\left(K_{0} \cdot x\right)$ and $\mu:(-\epsilon, \epsilon) \rightarrow X$ be a curve in $K_{0} \cdot x$ with $\mu(0)=x$ and $\mu^{\prime}(0)=W$. Then, since $f^{+}$is constant on $K_{0} \cdot x$, we have

$$
\left\langle\nabla f^{+}, W\right\rangle_{x}=\left\langle\nabla f^{+}, \mu^{\prime}(0)\right\rangle_{x}=\left.\frac{d}{d t} f^{+}(\mu(t))\right|_{t=0}=0
$$

Proposition 3.2. Let $\gamma: \mathbb{R} \rightarrow X$ be a maximal integral curve for $\nabla f^{+}$ beginning at a noncritical point $x \in X$, and let $k \in K_{0}$. Then $k \cdot \gamma$ is also a maximal integral curve for $\nabla f^{+}$, beginning at $k \cdot x$.

Proof. Applying Proposition 2.3, we identify $X$ with an $\operatorname{Ad}\left(G_{u}\right)$-orbit $\mathcal{O}$ in $\mathfrak{g}_{u}$. Let $W \in \mathfrak{g}_{u}$ and $(\cdot, \cdot)$ be the symmetric part of the Kähler form. In this setting $k \cdot \gamma$ means $\operatorname{Ad}_{k} \circ \gamma$, and it suffices to show $\left(\operatorname{Ad}_{k} \circ \gamma\right)^{\prime}(0)=$ $\nabla f^{+}(k \cdot x)$, or rather, that

$$
\left(\left(\operatorname{Ad}_{k} \circ \gamma\right)^{\prime}(0), \xi_{W}\right)_{k \cdot x}=\xi_{W} \cdot f^{+}(k \cdot x)
$$

Using the definition of $\nabla f^{+}$together with the invariance of $f^{+}$under $K_{0}$, we obtain

$$
\begin{aligned}
\left(\left(\operatorname{Ad}_{k} \circ \gamma\right)^{\prime}(0), \xi_{W}\right)_{k \cdot x} & =\left(\nabla f^{+}(x), \xi_{\operatorname{Ad}_{k^{-1}} W}\right)_{x} \\
& =\xi_{\mathrm{Ad}_{k^{-1}} W} \cdot f^{+}(x)=\xi_{W} \cdot f^{+}(k \cdot x)
\end{aligned}
$$

In view of Proposition 3.1 and Proposition 2.5, each maximal integral curve $\gamma(t)$ for $\nabla f^{+}$must be tangent to $G_{0^{-}}$and $K$-orbits while being orthogonal to $K_{0}$-orbits. Further, since the limiting values $\lim _{t \rightarrow \pm \infty} \gamma(t)$ lie in critical $K_{0}$-orbits, Proposition 3.2 indicates that if one of these limiting values lies in $K_{0} \cdot x_{0}$, then we do no harm by assuming the limiting value is $x_{0}$. The simplest candidate for a curve $\gamma(t)$ that might satisfy these conditions is

$$
\gamma(t)=\operatorname{Ad}\left(e^{s(t) Z}\right) \Upsilon
$$

where $s$ is a (nonzero) real-valued function and $Z \in \mathfrak{g}_{u}$ is identified via Proposition 2.6 with either an element of $T_{x_{0}}\left(G_{0} \cdot x_{0}\right)$ or $T_{x_{0}}\left(K \cdot x_{0}\right)$ that 
lies in the orthogonal complement of $T_{x_{0}}\left(K_{0} \cdot x_{0}\right)$. Specifically, in view of Proposition 2.6, we consider $Z \in \mathfrak{g}_{u}$ exactly when $Z$ lies in the set

$$
\mathcal{Z}=\left\{Z=Z_{\tilde{\mathfrak{r}}}+Z_{\mathfrak{r}} \in \mathfrak{g}_{u} \mid Z_{\tilde{\mathfrak{r}}} \in\left[\operatorname{ad}_{i \Upsilon}^{-1} \tilde{\mathfrak{r}}^{-\sigma}\right]_{\tilde{\mathfrak{r}}} \quad \text { or } \quad Z_{\tilde{\mathfrak{r}}} \in\left[\operatorname{ad}_{i \Upsilon}^{-1} \tilde{\mathfrak{r}}^{-\sigma \theta}\right]_{\tilde{\mathfrak{r}}}\right\}
$$

In the sequel, we show:

- For certain $Z \in \mathcal{Z}$ there exists $s(t)$ so that the curve $\gamma(t)$ given by (3.1) is a maximal integral curve for $\nabla f^{+}$. These curves correspond to Cayley transforms.

- If $X$ is a rank-one irreducible hermitian symmetric space then for every $Z \in \mathcal{Z}$ there exists $s(t)$ such that $\gamma$ is a maximal integral curve for $\nabla f^{+}$. These curves are pregeodesic in $X$.

\section{The differential equation}

Let $\gamma(t)=\operatorname{Ad}\left(e^{s(t) Z}\right) \Upsilon$ be as in (3.1). We find that $\gamma$ will be an integral curve for $\nabla f^{+}$if and only if the scaling function $s(t)$ satisfies a certain differential equation.

Lemma 4.1. Let $\gamma(t)$ be as in (3.1), and $t_{0} \in \mathbb{R}$. Then $\gamma^{\prime}\left(t_{0}\right)=\xi_{s^{\prime}(t) Z}\left(\gamma\left(t_{0}\right)\right)$.

Proof. Let $f: X \rightarrow \mathbb{C}$ be smooth. Appealing to the Taylor's theorem, we have

$$
\begin{aligned}
\left(\gamma^{\prime}\left(t_{0}\right) \cdot f\right)\left(\gamma\left(t_{0}\right)\right) & =\left.\frac{d}{d t}\right|_{t=t_{0}} f\left(\operatorname{Ad}\left(e^{s(t) Z}\right) \Upsilon\right) \\
& =\left.\frac{d}{d r}\right|_{r=0} f\left(\operatorname{Ad}\left(e^{s\left(t_{0}+r\right) Z}\right) \Upsilon\right) \\
& =\left.\frac{d}{d r}\right|_{r=0} f\left(\operatorname{Ad}\left(e^{\left(r s^{\prime}\left(t_{0}\right)+s\left(t_{0}\right)\right) Z}\right) \Upsilon\right) \\
& =\left.\frac{d}{d r}\right|_{r=0} f\left(\operatorname{Ad}\left(e^{r s^{\prime}\left(t_{0}\right) Z}\right) \gamma\left(t_{0}\right)\right) \\
& =\xi_{s^{\prime}\left(t_{0}\right) Z} \cdot f\left(\gamma\left(t_{0}\right)\right) .
\end{aligned}
$$

Therefore $\gamma^{\prime}\left(t_{0}\right)=\xi_{s^{\prime}\left(t_{0}\right) Z}\left(\gamma\left(t_{0}\right)\right)$.

On the other hand, (2.5) together with (2.2) tell us that

$$
\nabla f^{+}\left(\gamma\left(t_{0}\right)\right)=-2 J \xi_{\sigma \gamma\left(t_{0}\right)}\left(\gamma\left(t_{0}\right)\right)=\xi_{2 i\left(\left[\sigma \gamma\left(t_{0}\right)\right]_{\mathfrak{r}_{\gamma\left(t_{0}\right)}}-\left[\sigma \gamma\left(t_{0}\right)\right]_{\tilde{\mathfrak{r}}_{\gamma\left(t_{0}\right)}}\right)}
$$


Putting (4.1) together with Lemma 4.1, we see that $\gamma(t)$ is an integral curve for $\nabla f^{+}$if and only if

$$
s^{\prime}(t) Z \equiv 2 i\left(\left[\sigma \gamma\left(t_{0}\right)\right]_{\mathfrak{r}_{\gamma\left(t_{0}\right)}}-\left[\sigma \gamma\left(t_{0}\right)\right]_{\tilde{\mathfrak{r}}_{\gamma\left(t_{0}\right)}}\right) \bmod \left(\mathfrak{l}_{\gamma(t)}\right)
$$

for all $t \in \mathbb{R}$. We may simplify (4.2) as follows:

Proposition 4.2. Let $\gamma(t)$ be as in (3.1) with $Z \in \mathcal{Z}$. Then $\gamma(t)$ is an integral curve for $\nabla f^{+}$if and only if $s(t)$ satisfies

$$
s^{\prime}(t) Z=2 i\left(\left[\operatorname{Ad}\left(e^{-s Z} e^{\sigma s Z}\right) \sigma \Upsilon\right]_{\mathfrak{r}}-\left[\operatorname{Ad}\left(e^{-s Z} e^{\sigma s Z}\right) \sigma \Upsilon\right]_{\mathfrak{r}}\right),
$$

where $\mathfrak{r}$ denotes $\mathfrak{r}_{x_{0}}$.

Proof. Since $\sigma \circ \operatorname{Ad}_{g}=\operatorname{Ad}_{\sigma g} \circ \sigma$ for each $g \in G$, we have

$$
\begin{aligned}
{[\sigma \gamma(t)]_{\mathfrak{r}_{\gamma(t)}}=\left[\operatorname{Ad}\left(e^{\sigma s Z}\right) \sigma \Upsilon\right]_{\mathfrak{r}_{\gamma(t)}} } & =\left[\operatorname{Ad}\left(e^{s Z}\right) \operatorname{Ad}\left(e^{-s Z} e^{\sigma s Z}\right) \sigma \Upsilon\right]_{\mathfrak{r}_{\gamma(t)}} \\
& =\operatorname{Ad}\left(e^{s Z}\right)\left[\operatorname{Ad}\left(e^{-s Z} e^{\sigma s Z}\right) \sigma \Upsilon\right]_{\mathfrak{r}}
\end{aligned}
$$

and similarly $[\sigma \gamma(t)]_{\tilde{\mathfrak{r}}_{\gamma(t)}}=\operatorname{Ad}\left(e^{s Z}\right)\left[\operatorname{Ad}\left(e^{-s Z} e^{\sigma s Z}\right) \sigma \Upsilon\right]_{\tilde{\mathfrak{r}}}$. Therefore, (4.2) becomes

$$
\begin{aligned}
s^{\prime}(t) Z \equiv 2 i\left(\operatorname{Ad}\left(e^{s Z}\right)\right. & {\left[\operatorname{Ad}\left(e^{-s Z} e^{\sigma s Z}\right) \sigma \Upsilon\right]_{\mathfrak{r}} } \\
& \left.-\operatorname{Ad}\left(e^{s Z}\right)\left[\operatorname{Ad}\left(e^{-s Z} e^{\sigma s Z}\right) \sigma \Upsilon\right]_{\mathfrak{r}}\right) \bmod \left(\mathfrak{l}_{\gamma(t)}\right) .
\end{aligned}
$$

We then obtain the result by applying $\operatorname{Ad}\left(e^{-s Z}\right)$ on both sides of (4.3) and keeping in mind that $Z$ has no contribution from $\mathfrak{l}$.

\section{Cayley transforms}

Here we show that for $Z \in \mathcal{Z}$ that are built from a single root in $\Delta(\tilde{\mathfrak{r}}, \mathfrak{t})$ there exists a solution $s(t)$ for the differential equation given in Proposition 4.2. The corresponding integral curves are identified with Cayley transforms.

Theorem 5.1. Let $\alpha \in \Delta(\tilde{\mathfrak{r}}, \mathfrak{t})$. If $Z_{\alpha} \in\left(\mathfrak{g}^{\alpha} \cap \tilde{\mathfrak{r}}^{-\sigma}\right)$ or $Z_{\alpha} \in\left(\mathfrak{g}^{\alpha} \cap \tilde{\mathfrak{r}}^{-\sigma \theta}\right)$ then

(a) $Z=Z_{\alpha}+\theta Z_{\alpha} \in \mathcal{Z}$

(b) There exists a real-valued function $s(t)$ so that the curve $\gamma(t)=\mathrm{Ad}$ $\left(e^{s(t) Z}\right) \Upsilon$ is a maximal integral curve for $\nabla f^{+}$in $X$. 
(c) If $Z_{\alpha} \in\left(\mathfrak{g}^{\alpha} \cap \tilde{\mathfrak{r}}^{-\sigma}\right)$ then $\lim _{t \rightarrow \infty} \operatorname{Ad}\left(e^{s(t) Z}\right)$ is a Cayley transform, while if $Z_{\alpha} \in\left(\mathfrak{g}^{\alpha} \cap \tilde{\mathfrak{r}}^{-\sigma \theta}\right)$ then $\lim _{t \rightarrow-\infty} \operatorname{Ad}\left(e^{s(t) Z}\right)$ is a Cayley transform.

Proof. Since ad $i \Upsilon$ acts on $\mathfrak{g}^{\alpha}$ by a real scalar (Lemma 2.2), it follows that $\mathfrak{g}^{\alpha} \cap \tilde{\mathfrak{r}}^{-\sigma}=\mathfrak{g}^{\alpha} \cap \operatorname{ad}_{i \Upsilon}^{-1} \tilde{\mathfrak{r}}^{-\sigma}$ and $\mathfrak{g}^{\alpha} \cap \tilde{\mathfrak{r}}^{-\sigma \theta}=\mathfrak{g}^{\alpha} \cap \operatorname{ad}_{i \Upsilon}^{-1} \tilde{\mathfrak{r}}^{-\sigma \theta}$. This establishes part (a).

For (b) we set about examining the differential equation in Proposition 4.2. In either case listed above, one may produce $H_{\alpha} \in \mathfrak{t}$ such that

$$
\left[Z_{\alpha}, \theta Z_{\alpha}\right]=-H_{\alpha}, \quad\left[H_{\alpha}, Z_{\alpha}\right]=2 Z_{\alpha} \quad \text { and } \quad\left[H_{\alpha}, \theta Z_{\alpha}\right]=-2 \theta Z_{\alpha} .
$$

Using an infinite series expansion together with the bracket relations (5.1) and the fact that $\sigma Z=-Z$, we obtain

$$
\begin{aligned}
s^{\prime}(t)\left(Z_{\alpha}+\theta Z_{\alpha}\right) & =2 i\left(\left[\operatorname{Ad}\left(e^{-2 s Z}\right) \sigma \Upsilon\right]_{\mathfrak{r}}-\left[\operatorname{Ad}\left(e^{-2 s Z}\right) \sigma \Upsilon\right]_{\mathfrak{r}}\right) \\
& =2 i\left(\left[-\frac{\alpha(\sigma \Upsilon)}{2} \sin (4 s(t)) \theta Z_{\alpha}\right]+\left[-\frac{\alpha(\sigma \Upsilon)}{2} \sin (4 s(t)) Z_{\alpha}\right]\right) \\
& =-i \alpha(\sigma \Upsilon) \sin (4 s(t))\left(Z_{\alpha}+\theta Z_{\alpha}\right) .
\end{aligned}
$$

This differential equations yields gudermannian solutions

$$
s(t)=\frac{1}{2} \arctan \left(e^{-4 i \alpha(\sigma \Upsilon) t+C}\right),
$$

and consequently (b) follows from Proposition 4.2.

Finally, for item (c), we first observe that if $\mathfrak{g}^{\alpha} \cap \tilde{\mathfrak{r}}^{-\sigma}$ is nontrivial then $\alpha$ is a real root, $i \alpha(\sigma \Upsilon)<0$ (due to Lemma 2.2) and $\lim _{t \rightarrow \infty} s(t)=\pi / 4$ (due to (5.2)). Meanwhile if $\mathfrak{g}^{\alpha} \cap \tilde{\mathfrak{r}}^{-\sigma \theta}$ is nontrivial then $\alpha$ is a noncompact imaginary root, $i \alpha(\sigma \Upsilon)>0$ and $\lim _{t \rightarrow-\infty} s(t)=\pi / 4$. With these facts in hand, we may conclude ([6], p. 390) that the limiting cases, namely $\operatorname{Ad}\left(e^{(\pi / 4)\left(Z_{\alpha}+\theta Z_{\alpha}\right)}\right)$, are Cayley transforms.

\section{Solving the differential equation in the rank-one Hermitian symmetric case}

In the case that $X$ is an irreducible rank-one symmetric space (of compact type), we demonstrate that all the curves $\gamma(t)$ described in (3.1) are integral curves for $\nabla f^{+}$by showing that the differential equation in Proposition 4.2 possesses a solution for each $Z \in \mathcal{Z}$. In addition, we observe that these integral curves are pregeodesic in $X$. Throughout we assume that $G$ is a simply connected complex simple Lie group. 


\subsection{Flag manifolds and symmetric spaces}

Before addressing the differential equation in Proposition 4.2, we list some facts about hermitian symmetric spaces that will be useful as we proceed. These facts may be found, for example, in [3,11].

Proposition 6.1. If $X=G_{u} /\left(G_{u} \cap L\right)$ is a hermitian symmetric space then $\Delta(\mathfrak{r}, \mathfrak{t})$ contains exactly one simple root.

Proposition 6.1 immediately yields the following corollaries:

Corollary 6.2. Suppose $X=G_{u} /\left(G_{u} \cap L\right)$ is a hermitian symmetric space, $\beta$ is the unique simple root in $\Delta(\mathfrak{r}, \mathfrak{t})$ and $\alpha_{1}, \ldots, \alpha_{n}$ are the remaining simple roots in $\Delta(\mathfrak{g}, \mathfrak{t})$ (these simple roots span the $\mathfrak{l}$-roots). If $\alpha \in \Delta(\mathfrak{r}, \mathfrak{t})$ then $\alpha$ has the form

$$
\alpha=\beta+\sum_{j=1}^{n} k_{j} \alpha_{j},
$$

where each $k_{j}$ is a non-negative integer.

Corollary 6.3. If $X=G_{u} /\left(G_{u} \cap L\right)$ a hermitian symmetric space then $[\mathfrak{r}, \mathfrak{r}]=0,[\mathfrak{r}, \mathfrak{l}] \subset \mathfrak{r}$ and $[\mathfrak{r}, \tilde{\mathfrak{r}}] \subset \mathfrak{l}$.

\subsection{Solving the differential equation}

In a series of lemmas culminating in Theorem 6.8, we show that if $X$ is an irreducible hermitian symmetric of rank one and $Z \in \mathcal{Z}$, then the curve $\gamma$ given in (3.1) is a maximal integral curve for $\nabla f^{+}$.

Lemma 6.4. Let $Z=Z_{\tilde{\mathfrak{r}}}+Z_{\mathfrak{r}} \in \mathcal{Z}$.

(a) $\left[\operatorname{ad}_{i \Upsilon}^{-1} \tilde{\mathfrak{r}}^{-\sigma}\right]_{\mathfrak{r}}=\tilde{\mathfrak{r}}^{-\sigma}$ and $\left[\operatorname{add}_{i \Upsilon}^{-1} \tilde{\mathfrak{r}}^{-\sigma \theta}\right]_{\tilde{\mathfrak{r}}}=\tilde{\mathfrak{r}}^{-\sigma \theta}$.

(b) $\sigma Z=-Z$.

Proof. For (a), let $V \in \tilde{\mathfrak{r}}$ and write $V=V_{\alpha_{1}}+\cdots+V_{\alpha_{n}}$ where each $\alpha_{j} \in$ $\Delta(\tilde{\mathfrak{r}}, \mathfrak{t})$ and $V_{\alpha_{j}} \in \mathfrak{g}^{\alpha_{j}}$. By Corollary 6.2, each $\alpha_{j}$ has the form $\gamma+\beta_{j}$ where $-\gamma$ is the unique simple root in $\Delta(\mathfrak{r}, \mathfrak{t})$ and $\beta_{j}$ is the negative of a sum of simple roots in $\Delta(\mathfrak{l}, \mathfrak{t})$. (In fact, $\beta_{j} \in \Delta(\mathfrak{l}, \mathfrak{t})$ in case $X$ is of rank one.) 
Therefore, since $\Upsilon$ commutes with $\mathfrak{l}$ (Lemma 2.2), we have

$$
\begin{aligned}
\operatorname{add}_{i \Upsilon V} & =\left(\gamma+\beta_{1}\right)(i \Upsilon) V_{\alpha_{1}}+\cdots+\left(\gamma+\beta_{n}\right)(i \Upsilon) V_{\alpha_{n}} \\
& =\gamma(i \Upsilon) V_{\alpha_{1}}+\cdots+\gamma(i \Upsilon) V_{\alpha_{n}} \\
& =\gamma(i \Upsilon) V .
\end{aligned}
$$

Further, by Lemma 2.2, $\gamma(i \Upsilon)>0$. Therefore, $\operatorname{ad}_{i \Upsilon}$ acts on $\tilde{\mathfrak{r}}$ by a nonzero real scalar, so ad ${ }_{i \Upsilon}^{-1}$ preserves both $\tilde{\mathfrak{r}}^{-\sigma}$ and $\tilde{\mathfrak{r}}^{-\sigma \theta}$.

Part (b) follows immediately from part (a).

Lemma 6.5. Let $Z_{\mathfrak{r}} \in \tilde{\mathfrak{r}}, Z_{\mathfrak{r}}=\theta Z_{\mathfrak{r}}$ and $T: \mathfrak{g} \rightarrow \mathfrak{g}$ be defined by $T_{Z_{\mathfrak{r}}}=\operatorname{ad}_{Z_{\mathfrak{r}}} \circ$ $\operatorname{ad}_{Z_{\tilde{\mathfrak{r}}}}$. For each whole number $n$ we have

$$
\left[\left[Z_{\mathfrak{r}}, Z_{\tilde{\mathfrak{r}}}\right], T_{Z_{\mathfrak{r}}}^{n}\left(\left[Z_{\mathfrak{r}}, Z_{\tilde{\mathfrak{r}}}\right]\right)\right]=0
$$

(Here $T^{0}$ is the identity mapping.)

Proof. Let $\beta$ denote the unique simple root in $\Delta(\mathfrak{r}, \mathfrak{t})$, and $Z_{\beta}$ a nonzero element of $\mathfrak{g}^{\beta}$. Then

$$
\left\{\left[V, Z_{\beta}\right] \mid V \in \mathfrak{t}_{u}+\sum_{\alpha \in \Delta^{+}(\mathfrak{l}, \mathfrak{t})} \mathfrak{g}^{\alpha}\right\}
$$

is a subspace of $\mathfrak{r}$ of real co-dimension one. Further, since mixed sign combinations of simple roots never yield a root, we have $\left[\theta \mathrm{V}, Z_{\beta}\right]=0$ whenever $V \in \sum_{\alpha \in \Delta^{+}(\mathfrak{l}, \mathfrak{t})} \mathfrak{g}^{\alpha}$, and hence $\left[V, Z_{\beta}\right]=\left[V+\theta V, Z_{\beta}\right]$ in this case. We may then conclude $\operatorname{ad}_{\mathfrak{n} \mathfrak{g}_{u}}\left(Z_{\beta}\right)$ is a real co-dimension one subspace of $\mathfrak{r}$. Therefore $\operatorname{Ad}_{G_{u} \cap L} Z_{\beta}$ (a sphere in $\mathfrak{r}$ ) must also be of co-dimension one, and so $\operatorname{Ad}_{G_{u} \cap L}\left(\mathbb{C} Z_{\beta}\right)$ is all of $\mathfrak{r}$.

Now, suppose $Z_{\mathfrak{r}}=\operatorname{Ad}_{g} c Z_{\beta}$ for some $g \in G_{u} \cap L$ and $c \in \mathbb{C}$. Then $Z_{\mathfrak{r}}=$ $\operatorname{Ad}_{g} \theta\left(c Z_{\beta}\right)$, and since $\operatorname{Ad}_{g}$ is a Lie algebra homomorphism we obtain

$$
\left[\left[Z_{\mathfrak{r}}, Z_{\mathfrak{r}}\right], T^{n}\left(\left[Z_{\mathfrak{r}}, Z_{\mathfrak{r}}\right]\right)\right]=\operatorname{Ad}_{g}\left[\left[c Z_{\beta}, \theta c Z_{\beta}\right], T_{c Z_{\beta}}^{n}\left(\left[c Z_{\beta}, \theta c Z_{\beta}\right]\right)\right] .
$$

Since both $\left[c Z_{\beta}, \theta c Z_{\beta}\right]$ and $T_{c Z_{\beta}}^{n}\left(\left[c Z_{\beta}, \theta c Z_{\beta}\right]\right)$ lie in $\mathfrak{t}$, the right-hand side of (6.1) is zero, thus giving the result.

Lemma 6.6. Let $Z_{\tilde{\mathfrak{r}}} \in \tilde{\mathfrak{r}}, Z_{\mathfrak{r}}=\theta Z_{\tilde{\mathfrak{r}}}$ and $Z=Z_{\mathfrak{r}}+Z_{\tilde{\mathfrak{r}}} \in \mathfrak{g}_{u}$. Then

$$
\left[Z_{\tilde{\mathfrak{r}}},\left(\operatorname{Ad}_{e^{z}} \Upsilon\right)_{\mathfrak{r}}\right]=\left[Z_{\mathfrak{r}},\left(\operatorname{Ad}_{e^{z}} \Upsilon\right)_{\tilde{\mathfrak{r}}}\right]
$$


Proof. We first examine $\operatorname{Ad}_{e^{z}} \Upsilon$. Recall that

$$
\operatorname{Ad}_{e^{z}} \Upsilon=e^{\operatorname{ad}_{z}} \Upsilon=\sum_{n=0}^{\infty} \frac{1}{n !}\left(\operatorname{ad}_{Z}\right)^{n} \Upsilon
$$

Using Corollary 6.3, Lemma 6.5 and the fact that $\left[Z_{\mathfrak{r}}, \Upsilon\right]=c Z_{\mathfrak{r}}$ for some $c \in i \mathbb{R}$ (see proof of Lemma 6.4), the series on the right-hand side of (6.2) may be expressed as $\sum_{n=1}^{\infty} V_{n}$, with $V_{1}=\Upsilon, V_{2}=c\left(Z_{\mathfrak{r}}-Z_{\mathfrak{r}}\right)$ and for $k \geq 1$

$$
V_{2(k+1)}=a_{2(k+1)}\left(\operatorname{ad}_{Z_{\mathfrak{r}}} T_{Z_{\mathfrak{r}}}^{k-1}\left(\left[Z_{\mathfrak{r}}, Z_{\tilde{\mathfrak{r}}}\right]\right)+\operatorname{ad}_{Z_{\mathfrak{r}}} T_{Z_{\mathfrak{r}}}^{k-1}\left(\left[Z_{\mathfrak{r}}, Z_{\tilde{\mathfrak{r}}}\right]\right)\right)
$$

and

$$
V_{2 k+1}=a_{2 k+1} T_{Z_{\mathfrak{r}}}^{k-1}\left(\left[Z_{\mathfrak{r}}, Z_{\tilde{\mathfrak{r}}}\right]\right)
$$

where $a_{n} \in i \mathbb{R}$ for $n>2$. Therefore

$$
\left(\operatorname{Ad}_{e^{z}} \Upsilon\right)_{\mathfrak{r}}=c Z_{\mathfrak{r}}+\sum_{k=1}^{\infty} a_{2(k+1)} \operatorname{ad}_{Z_{\mathfrak{r}}} T_{Z_{\mathfrak{r}}}^{k-1}\left(\left[Z_{\mathfrak{r}}, Z_{\tilde{\mathfrak{r}}}\right]\right)
$$

while

$$
\left(\operatorname{Ad}_{e^{Z}} \Upsilon\right)_{\tilde{\mathfrak{r}}}=-c Z_{\tilde{\mathfrak{r}}}+\sum_{k=1}^{\infty} a_{2(k+1)} \operatorname{ad}_{Z_{\mathfrak{r}}} T_{Z_{\mathfrak{r}}}^{k-1}\left(\left[Z_{\mathfrak{r}}, Z_{\tilde{\mathfrak{r}}}\right]\right)
$$

From (6.5) and (6.6), we have

$$
\left[Z_{\tilde{\mathfrak{r}}},\left(\operatorname{Ad}_{e^{z}} \Upsilon\right)_{\mathfrak{r}}\right]=\left[Z_{\tilde{\mathfrak{r}}}, c Z_{\mathfrak{r}}\right]+\sum_{k=1}^{\infty} a_{2(k+1)} \operatorname{ad}_{Z_{\mathfrak{r}}} \operatorname{ad}_{Z_{\mathfrak{r}}} T_{Z_{\mathfrak{r}}}^{k-1}\left(\left[Z_{\mathfrak{r}}, Z_{\tilde{\mathfrak{r}}}\right]\right)
$$

and

$$
\left[Z_{\mathfrak{r}},\left(\operatorname{Ad}_{e^{z}} \Upsilon\right)_{\tilde{\mathfrak{r}}}\right]=\left[Z_{\mathfrak{r}},-c Z_{\tilde{\mathfrak{r}}}\right]+\sum_{k=1}^{\infty} a_{2(k+1)} \operatorname{ad}_{Z_{\mathfrak{r}}} \operatorname{ad}_{Z_{\mathfrak{r}}} T_{Z_{\mathfrak{r}}}^{k-1}\left(\left[Z_{\mathfrak{r}}, Z_{\mathfrak{r}}\right]\right)
$$

Clearly the first terms of (6.7) and (6.8) agree, and the remaining summands agree term by term by applying the Jacobi identity followed by Lemma 6.5. 
Lemma 6.7. Let $Z_{\tilde{\mathfrak{r}}} \in \tilde{\mathfrak{r}} Z_{\mathfrak{r}}=\theta Z_{\tilde{\mathfrak{r}}}$ and $Z=Z_{\mathfrak{r}}+Z_{\tilde{\mathfrak{r}}} \in \mathfrak{g}_{u}$.

(a) If $Z_{\tilde{\mathfrak{r}}} \in \tilde{\mathfrak{r}}^{-\sigma}$, then $\left(\sigma \operatorname{Ad}_{e^{z}} \Upsilon\right)_{\tilde{\mathfrak{r}}}=\sigma\left(\left(\operatorname{Ad}_{e^{z}} \Upsilon\right)_{\mathfrak{r}}\right)$.

(b) If $Z_{\tilde{\mathfrak{r}}} \in \tilde{\mathfrak{r}}^{-\sigma \theta}$, then $\left(\sigma \operatorname{Ad}_{e^{z}} \Upsilon\right)_{\tilde{\mathfrak{r}}}=\sigma\left(\left(\operatorname{Ad}_{e^{z}} \Upsilon\right)_{\mathfrak{r}}\right)$.

Proof. For part (a), the hypothesis together with (6.6) and the fact that $c, a_{n} \in i \mathbb{R}$ yields

$$
\begin{aligned}
\sigma\left(\left(\operatorname{Ad}_{e^{z}} \Upsilon\right)_{\tilde{\mathfrak{r}}}\right) & =\sigma\left(-c Z_{\tilde{\mathfrak{r}}}+\sum_{k=1}^{\infty} a_{2(k+1)} \operatorname{ad}_{Z_{\tilde{\mathfrak{r}}}} T_{Z_{\mathfrak{r}}}^{k-1}\left(\left[Z_{\mathfrak{r}}, Z_{\tilde{\mathfrak{r}}}\right]\right)\right) \\
& =-c Z_{\tilde{\mathfrak{r}}}+\sum_{k=1}^{\infty} a_{2(k+1)} \operatorname{ad}_{Z_{\mathfrak{r}}} T_{Z_{\mathfrak{r}}}^{k-1}\left(\left[Z_{\mathfrak{r}}, Z_{\tilde{\mathfrak{r}}}\right]\right) .
\end{aligned}
$$

Meanwhile, from the proof of Lemma 6.6 we have $\sigma \operatorname{Ad}_{e^{z}} \Upsilon=\sum_{n=1}^{\infty} \sigma V_{n}$, where $\sigma V_{1}=\sigma \Upsilon, \sigma V_{2}=c\left(Z_{\mathfrak{r}}-Z_{\mathfrak{r}}\right)$, and for $k \geq 1$

$$
\sigma V_{2(k+1)}=a_{2(k+1)}\left(\operatorname{ad}_{Z_{\mathfrak{r}}} T_{Z_{\mathfrak{r}}}^{k-1}\left(\left[Z_{\mathfrak{r}}, Z_{\tilde{\mathfrak{r}}}\right]\right)+\operatorname{ad}_{Z_{\mathfrak{r}}} T_{Z_{\mathfrak{r}}}^{k-1}\left(\left[Z_{\mathfrak{r}}, Z_{\tilde{\mathfrak{r}}}\right]\right)\right)
$$

and

$$
\sigma V_{2 k+1}=-a_{2 k+1} T_{Z_{\mathfrak{r}}}^{k-1}\left(\left[Z_{\mathfrak{r}}, Z_{\tilde{\mathfrak{r}}}\right]\right)
$$

Therefore, upon extracting the $\tilde{\mathfrak{r}}$ part we have

$$
\left(\sigma \operatorname{Ad}_{e^{z}} \Upsilon\right)_{\tilde{\mathfrak{r}}}=-c Z_{\tilde{\mathfrak{r}}}+\sum_{k=1}^{\infty} a_{2(k+1)} \operatorname{ad}_{Z_{\tilde{\mathfrak{r}}}} T_{Z_{\mathfrak{r}}}^{k-1}\left(\left[Z_{\mathfrak{r}}, Z_{\tilde{\mathfrak{r}}}\right]\right)
$$

and we conclude that $\left(\sigma \operatorname{Ad}_{e^{z}} \Upsilon\right)_{\tilde{\mathfrak{r}}}=\sigma\left(\left(\operatorname{Ad}_{e^{z}} \Upsilon\right)_{\tilde{\mathfrak{r}}}\right)$.

The proof for part (b) is similar to that for part (a). (In part (b) one needs the fact that $T_{Z_{\mathfrak{r}}}^{k-1}\left(\left[Z_{\mathfrak{r}}, Z_{\mathfrak{r}}\right]\right)=T_{Z_{\mathfrak{r}}}^{k-1}\left(\left[Z_{\mathfrak{r}}, Z_{\mathfrak{r}}\right]\right.$.) This follows by induction using Lemma 6.5.)

Theorem 6.8. If $Z=Z_{\mathfrak{r}}+Z_{\mathfrak{r}} \in \mathcal{Z}$ and $\gamma(t)$ is the corresponding curve in $X$ described in (3.1), then $\gamma(t)$ is a maximal integral curve for $\nabla f^{+}$.

Proof. For $Z \in \mathcal{Z}$, let $W_{Z}(t) \in(\mathfrak{r}+\tilde{\mathfrak{r}})_{u} \subset \mathfrak{g}_{u}$ denote the right-hand side of the differential equation in Proposition 4.2. Observe that the differential equation possesses a solution if and only if $W_{Z}(t)$ is a real scalar multiple of 
$Z$ for each $t \in \mathbb{R}$. In case $X$ is symmetric of rank one, $W_{Z}(t)$ is a real scalar multiple of $Z$ if and only if $\left[Z, W_{Z}(t)\right]=0$. So, it remains for us to show that $\left[Z, W_{Z}(t)\right]=0$.

To begin, Lemma 6.4 implies that $W_{Z}(t)$ simplifies to

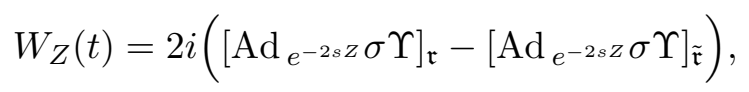

and from this it follows that

$$
\left[Z, W_{Z}(t)\right]=0 \Longleftrightarrow\left[Z_{\mathfrak{r}},\left(\operatorname{Ad}_{e^{-2 s} z \sigma} \sigma\right)_{\mathfrak{r}}\right]=\left[Z_{\tilde{\mathfrak{r}}},\left(\operatorname{Ad}_{\left.\left.e^{-2 s z} \sigma \Upsilon\right)_{\mathfrak{r}}\right]}\right.\right.
$$

We finish the proof by verifying the right-hand Equation in (6.11). By Lemma 6.4, there are two cases to consider. First, suppose that $Z_{\mathfrak{r}} \in \tilde{\mathfrak{r}}^{-\sigma}$. Applying Lemmas 6.6 and 6.7, we have

$$
\begin{aligned}
& {\left[Z_{\mathfrak{r}},\left(\operatorname{Ad}_{e^{-2 s} z} \sigma \Upsilon\right)_{\mathfrak{r}}\right]=\left[Z_{\tilde{\mathfrak{r}}},\left(\sigma\left(\operatorname{Ad}_{e^{2 s z}} \Upsilon\right)\right)_{\mathfrak{r}}\right]=\left[Z_{\mathfrak{r}}, \sigma\left(\left(\operatorname{Ad}_{e^{2 s z}} \Upsilon\right)_{\mathfrak{r}}\right)\right]} \\
& =\sigma\left[-Z_{\tilde{\mathfrak{r}}},\left(\operatorname{Ad}_{e^{2 s z}} \Upsilon\right)_{\mathfrak{r}}\right]=\sigma\left[-Z_{\mathfrak{r}},\left(\operatorname{Ad}_{e^{2 s Z}} \Upsilon\right)_{\tilde{\mathfrak{r}}}\right] \\
& =\left[Z_{\mathfrak{r}}, \sigma\left(\left(\operatorname{Ad}_{e^{2 s Z}} \Upsilon\right)_{\tilde{\mathfrak{r}}}\right)\right]=\left[Z_{\mathfrak{r}},\left(\sigma\left(\operatorname{Ad}_{e^{2 s Z}} \Upsilon\right)\right)_{\tilde{\mathfrak{r}}}\right] \\
& =\left[Z_{\mathfrak{r}},\left(\operatorname{Ad}_{\left.e^{-2 s z} \sigma \Upsilon\right)}\right) \tilde{\mathfrak{r}}\right] .
\end{aligned}
$$

Similarly, if $Z_{\tilde{\mathfrak{r}}} \in \tilde{\mathfrak{r}}^{-\sigma \theta}$, then

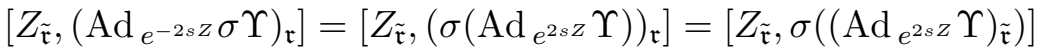

$$
\begin{aligned}
& =\sigma\left[-Z_{\mathfrak{r}},\left(\operatorname{Ad}_{e^{2 s z}} \Upsilon\right)_{\tilde{\mathfrak{r}}}\right]=\sigma\left[-Z_{\tilde{r}},\left(\operatorname{Ad}_{e^{2 s Z}} \Upsilon\right)_{\mathfrak{r}}\right] \\
& =\left[Z_{\mathfrak{r}}, \sigma\left(\left(\operatorname{Ad}_{e^{2 s Z}} \Upsilon\right)_{\mathfrak{r}}\right)\right]=\left[Z_{\mathfrak{r}},\left(\sigma\left(\operatorname{Ad}_{e^{2 s Z}} \Upsilon\right)\right)_{\mathfrak{r}}\right] \\
& =\left[Z_{\mathfrak{r}},\left(\operatorname{Ad}_{\left.e^{-2 s} Z \sigma \Upsilon\right)}\right) \tilde{\mathfrak{r}}\right] .
\end{aligned}
$$

Recall (see, for example, [9]) that if $G / H$ is a naturally reductive homogeneous space with subspace with $\mathfrak{m}$ the associated complementary subspace to $\mathfrak{h}$ in $\mathfrak{g}$, then geodesics starting at $o=e H$ have the form

$$
\Gamma_{d \pi X}(t)=\alpha(t) \cdot o=\pi \alpha(t),
$$

where $\alpha(t)$ is the one-parameter subgroup in $G$ corresponding to $X \in \mathfrak{m} \subset \mathfrak{g}$, and $\pi: G \rightarrow G / H$. Specializing to our situation (with $o=\Upsilon$ and the adjoint action of $G_{u}$ on $X$ ) we conclude that geodesics in $X$ starting at $\Upsilon$ have the 
form $\operatorname{Ad}\left(e^{t Z}\right) \Upsilon$ where $Z \in(\mathfrak{r}+\tilde{\mathfrak{r}})$. Therefore, upon re-parameterization and Theorem 6.8 we have:

Corollary 6.9. For each $Z \in \mathcal{Z}$ the integral curves $\gamma$ for $\nabla f^{+}$given in (3.1) are pre-geodesic in $X$.

\section{Acknowledgment}

I extend thanks to Ralph Bremigan, my friend and colleague, for his help, encouragement and understanding throughout the years.

\section{References}

[1] M. Audin, The topology of torus actions on symplectic manifolds, Birkhäuser, Boston-Basel-Berlin, 1991.

[2] R. Bremigan and J. Lorch, Orbit duality for flag manifolds, Manuscripta Math. 109 (2002), 233-261.

[3] D. Drucker, Exceptional Lie algebras and the structure of hermitian symmetric spaces, Mem. Am. Math. Soc. 16(1) (1978), 1-207.

[4] G. Fels, A. Huckleberry and J. Wolf, Cycle spaces of flag domains, Birkhäuser, Boston, 2006.

[5] F. Kirwan, Cohomology of quotients in symplectic and algebraic geometry, Mathematical notes, vol. 31, Princeton University Press, Princeton, 1984.

[6] A. Knapp, Lie groups beyond an introduction, 2 edn, Birkhauser, 1996.

[7] T. Matsuki, The orbits of affine symmetric spaces under the action of parabolic subgroups, J. Math. Soc. Jap. 31 (1979), 331-357.

[8] I. Mirkovic, T. Uzawa, and K. Vilonen, Matsuki correspondence for sheaves, Invent. Math. 109 (1992), 231-245.

[9] B. O'Niell, Semi-Riemannian geometry and applications to relativity, Academic Press, San Diego, 1983.

[10] T. Uzawa, Invariant hyperfunction sections of line bundles, Thesis, Yale University, 1990. 
[11] J. Wolf, Flag manifolds and representation theory, in Geometry and representation theory of real and $p$-adic groups (Cordoba, 1995), Prog. Math., vol. 158, Birkhäuser, Boston, 1998, 273-323.

[12] R. Zierau, Representations in Dolbeault cohomology, in eds. Representation theory of Lie groups, J. Adams, D. Vogan, IAS/Park City Mathematics Series, vol. 8, American Mathematical Society, Providence, Rhode Island, 2000, 89-146.

Department of Mathematical Sciences

Ball State University

MUNCIE IN 47306-0490 USA

E-mail address: jlorch@bsu.edu

Received April 12, 2006 